\title{
On a conjecture about Dirac's delta representation using q-exponentials
}

\author{
A. Chevreuil, ${ }^{1}$ A. Plastino, ${ }^{2}$ and C. Vignat ${ }^{3, a)}$ \\ ${ }^{1}$ L.I.G.M., Université Paris-Est-ESIEE, 5 Bd Descartes, 77454 Marne la Vallée cedex, \\ France \\ ${ }^{2}$ C.C.T.-Conicet, National University La Plata, C.C. 727, 1900 La Plata, Argentina \\ ${ }^{3}$ L.S.S., Supelec, 3 rue Joliot-Curie, 91192 Gif-sur-Yvette cedex, France
}

(Received 21 June 2010; accepted 22 July 2010; published online 1 September 2010)

A new representation of Dirac's delta distribution, based on the so-called $q$-exponentials, has been recently conjectured. We prove here that this conjecture is indeed valid. () 2010 American Institute of Physics. [doi:10.1063/1.3478886]

\section{INTRODUCTION}

Tsallis and Jauregui ${ }^{7}$ recently conjectured a representation of the Dirac delta distribution, which they call $\delta_{q}(x)$, based on $q$-exponential functions. However, they could not prove their conjecture and used numerical experiments that suggest its validity. In this note, we provide a rigorous mathematical approach to this problem and prove their conjecture by recourse to the notion of superstatistics.

\section{II. q-EXPONENTIALS AND SUPERSTATISTICS}

Statistical mechanics' most notorious and renowned probability distribution is that deduced by Gibbs for the canonical ensemble, ${ }^{1,2}$ usually referred to as the Boltzmann-Gibbs equilibrium distribution,

$$
p_{G}(i)=\frac{\exp \left(-\beta E_{i}\right)}{Z_{B G}}
$$

with $E_{i}$ the energy of the microstate labeled by $i, \beta=1 / k_{B} T$ the inverse temperature, $k_{B}$ the Boltzmann constant, and $Z_{B G}$ the partition function. The exponential term $F_{B G}=\exp (-\beta E)$ is called the Boltzmann-Gibbs factor. Recently Beck and Cohen ${ }^{3}$ advanced a generalization, called superstatistics, of this $\mathrm{BG}$ factor, assuming that the inverse temperature $\beta$ is a stochastic variable. The generalized statistical factor $F_{G S}$ is thus obtained as the multiplicative convolution,

$$
F_{G S}=\int_{0}^{\infty} \frac{d \beta}{\beta} f(\beta) \exp (-\beta E),
$$

where $f(\beta)$ is the density probability of the inverse temperature.

As stated above, $\beta$ is the inverse temperature, but the integration variable may also be any convenient intensive parameter. Superstatistics, meaning "superposition of statistics," takes into account fluctuations of such intensive parameters.

Beck and Cohen also show that if $f(\beta)$ is a Gamma distribution, nonextensive thermostatistics is obtained, which is of interest because this thermostatistics is today a very active field, with applications to several scientific disciplines. ${ }^{4-6}$ In working in a nonextensive framework, one has

${ }^{a)}$ Electronic mail: vignat@univ-mlv.fr. 
to deal with power-law distributions, which are certainly ubiquitous in physics (critical phenomena are just a conspicuous example ${ }^{8}$. Indeed, it is well known that power-law distributions arise when maximizing Tsallis' information measure,

$$
H_{q}(f)=\frac{1}{q-1}\left(1-\int_{-\infty}^{+\infty} f(x)^{q} d x\right),
$$

subject to appropriate constraints, where $q \neq 1$ is a real positive parameter called the nonextensivity index. More precisely, in the case of the canonical distribution, there is only one constraint, the energy $E$, i.e., $\left\langle X^{2}\right\rangle=E>0$, and the equilibrium canonical distribution writes in the case $q>1$,

$$
f_{q}(x)=\frac{1}{Z_{q}}\left(1-(1-q) \beta_{q} x^{2}\right)^{1 / 1-q},
$$

where $\beta_{q}$ and $Z_{q}$ stand for the nonextensive counterparts of $\beta$ and $Z_{B G}$ above.

In the rest of this paper, we will assume as in Ref. 7 that $1<q<2$.

Let us choose the branch cut $(-\infty,-1 / 1-q]$ along the negative real axis and define the $q$-exponential function for $z \in \mathbb{C} \backslash(-\infty,-1 / 1-q]$ as

$$
e_{q}(z)=(1+(1-q) z)^{1 / 1-q} .
$$

This allows us to rewrite the equilibrium distribution in the more natural way,

$$
f_{q}(x)=\frac{1}{Z_{q}} e_{q}\left(-\beta x^{2}\right) .
$$

It is a classical result that as $q \rightarrow 1^{+}$, Tsallis entropy reduces to Shannon entropy,

$$
H_{1}(f)=-\int_{-\infty}^{+\infty} f(x) \log f(x) .
$$

Accordingly, the $q$-exponential function $e_{q}(x)$ converges pointwise to the usual exponential function $e^{x}$.

\section{PROOF OF JAUREGUI-TSALLIS CONJECTURE}

\section{A. Definitions and notation}

Recall the formula

$$
\delta(t)=\frac{1}{2 \pi} \int_{\mathrm{R}} e^{-l u t} d u .
$$

We intend to provide a generalization of this relation; namely, we prove the following representation conjectured by Tsallis et al. assuming $1<q<2$ :

$$
\delta(t)=\frac{1}{c_{q}} \int_{\mathbb{R}} e_{q}(-\imath u t) d u
$$

for some constant $c_{q}$. We begin by recalling the mathematical meaning of (6).

Definition 1: A function $\varphi$ is called rapidly decreasing if $\varphi$ is $\mathcal{C}^{\infty}$ and if for all integers $k, \ell$,

$$
\lim _{x \rightarrow \pm \infty} x^{k} \varphi^{(\ell)}(x)=0
$$

Let $\mathcal{S}$ be the set of the rapidly decreasing functions on $\mathbb{R}$ and by $\mathcal{S}^{\prime}$ the set of the continuous linear functionals over $\mathcal{S}$.

We know from Rudin (Ref. 9, p. 184, Theorem 7.4) the following. 
Proposition 1: If $\varphi \in \mathcal{S}$, its Fourier transform $\mathcal{F}(\varphi)$ exists and belongs to $\mathcal{S}$. More precisely, the Fourier transform $\mathcal{F}$ is a continuous linear mapping of $\mathcal{S}$ into $\mathcal{S}$.

In the sequel, we use the notation $\hat{\varphi}=\mathcal{F}(\varphi)$.

Definition 2: Let now $f$ be a bounded measurable function. ${ }^{11}$ We let $T_{f}$ be the linear continuous mapping,

$$
\forall \varphi \in \mathcal{S}\left\langle T_{f}, \varphi\right\rangle=\int f(t) \varphi(t) d t
$$

\section{B. Proofs}

In order to prove the usual representation (6), we simply have to show that for all $\varphi \in \mathcal{S}$,

$$
\int d u\left\langle T_{\left.e^{-u u t}, \varphi\right\rangle}=2 \pi \varphi(0)\right.
$$

Of course $\left\langle T_{e^{-l u t}}, \varphi\right\rangle=\hat{\varphi}(u)$. Hence the result.

We now turn to the proof of (7). In this respect, let us pick a $\varphi \in \mathcal{S}$. We have

$$
\left\langle T_{e_{q}(-l u t)}, \varphi\right\rangle=\int e_{q}(-\imath u t) \varphi(t) d t=\int E_{W} e^{-l u t(q-1) W} \varphi(t) d t,
$$

where we have used the equality

$$
e_{q}(-\imath u t)=E_{W} e^{-\imath u t(q-1) W}
$$

Here

$$
E_{W} g(W) \triangleq \frac{1}{\Gamma\left(\frac{1}{q-1}\right)} \int_{0}^{+\infty} g(w) e^{-w} w^{(1 / q-1)-1} d w
$$

is the expectation of $g(W)$, where $W$ is a Gamma distributed random variable with shape parameter $1 / q-1$ and $g$ some function such that the above definition makes sense.

We note that (8) expresses the fundamental principle of the superstatistical theory.

On the other hand, we have

$$
\frac{1}{\Gamma\left(\frac{1}{q-1}\right)} \iint e^{-w} w^{(1 / q-1)-1}|\varphi(t)| d t d w \leq \int|\varphi(t)| d t .
$$

As obviously $\varphi$ is summable, we can apply the Fubini-Lebesgue theorem and we obtain

$$
\begin{aligned}
\left\langle T_{e_{q}(-l u t)}, \varphi\right\rangle & =E_{W} \int e^{-l u t(q-1) W} \varphi(t) d t=E_{W} \hat{\varphi}(u(q-1) W) \\
& =\frac{1}{\Gamma\left(\frac{1}{q-1}\right)} \int e^{-w} w^{(1 / q-1)-1} \hat{\varphi}(u(q-1) w) d w .
\end{aligned}
$$

Now, consider

$$
\int_{\mathrm{R}}\left\langle T_{e_{q}(-l u t)}, \varphi\right\rangle d u
$$

As $q<2$, we have, by the change of variable $u \mapsto v=u(q-1) w$, 


$$
\int d w \int\left|e^{-w} w^{(1 / q-1)-1} \hat{\varphi}(u(q-1) w)\right| d u=\frac{\int|\hat{\varphi}(v)| d v}{q-1} \int e^{-w} w^{(1 / q-1)-2} d w<\infty,
$$

since, by Proposition 1, $\hat{\varphi} \in \mathcal{S}$. Thanks to the Fubini-Lebesgue theorem, we deduce that

$$
\int_{\mathbb{R}}\left\langle T_{e_{q}(-u t)}, \varphi\right\rangle d u=\frac{1}{\Gamma\left(\frac{1}{q-1}\right)} \frac{\int \hat{\varphi}(v) d v}{q-1} \int e^{-w} w^{(1 / q-1)-2} d w=\frac{\Gamma\left(\frac{1}{q-1}-1\right)}{(q-1) \Gamma\left(\frac{1}{q-1}\right)} 2 \pi \varphi(0) .
$$

We have proved the result with

$$
c_{q}=\frac{2 \pi}{2-q} .
$$

\section{REMARKS ON JAUREGUI-TSALLIS APPROACH}

In their approach, ${ }^{7}$ the authors chose an empirical approach starting from the usual $q=1$ case: expressing the Dirac delta as the limit,

$$
\delta(x)=\frac{1}{c_{1} \lim _{L \rightarrow+\infty}} \int_{-L}^{+L} e^{-i k x} d k=\frac{2}{c_{1}} \lim \frac{\sin (L x)}{x},
$$

the normalization constant $c_{1}$ can be obtained formally as

$$
c_{1}=2 \lim _{L \rightarrow+\infty} \int_{-\infty}^{+\infty} \frac{\sin (L x)}{x} d x=2 \pi .
$$

The extension to $q$-exponentials reads

$$
\delta_{q}(x)=\frac{2}{(2-q) c_{q} L \rightarrow+\infty} \lim _{L} \frac{\sin \left(\frac{2-q}{q-1} \arctan ((q-1) L)\right)}{x\left(1+(q-1) L^{2} x^{2}\right)^{2-q / 2(q-1)}},
$$

so that the normalization constant $c_{q}$ can be obtained formally as

$$
c_{q}=\frac{2}{2-q} \lim _{L \rightarrow+\infty} \int_{-\infty}^{+\infty} \frac{\sin \left(\frac{2-q}{q-1} \arctan ((q-1) L)\right)}{x\left(1+(q-1) L^{2} x^{2}\right)^{2-q / 2(q-1)}} d x .
$$

This integral can be equivalently expressed using the change of variable $z=\tan \theta$ as

$$
I_{q} \triangleq 2 \int_{0}^{\frac{\pi}{2}} \frac{\sin \left(\frac{2-q}{q-1} \theta\right)(\cos \theta)^{2-q /(q-1)-1}}{\sin \theta} d \theta .
$$

The authors then evaluate the integral $I_{q}$ for a finite number of rational values of the parameter $q \in] 1,2\left[\right.$ only, for which the symbolic computation software MAPLE gives the value $I_{q}=\pi / 2$. However, this approach can be circumvented given the fact that the integral $I_{q}$ can be found in Ref. 10 (p. 3.638.3) with the value $\left.I_{q}=\pi / 2 \forall q \in\right] 1,2$ [ so that, following the above mentioned empirical approach used in Ref. 7, it can be deduced that $c_{q}=2 \pi / 2-q$. This allows us to confirm the correctness of the two representations of the number $\pi$ deduced from this result in Ref. 7 [Eqs. (23) and (25)]. 


\section{CONCLUSION}

We have proved that the representation of the Dirac delta distribution (7) using $q$-exponential functions, as conjectured by Tsallis et al., is valid. In particular, (i) we compute the exact normalization constant in the representation of the Dirac delta and (ii) we explicit the set of functions, namely, the set of rapidly decreasing functions defined in Definition 1, for which this distribution acts as the Dirac delta.

${ }^{1}$ F. Reif, Statistical and Thermal Physics (McGraw-Hill, New York, 1965); R. K. Pathria, Statistical Mechanics (Pergamon, Exeter, 1993).

${ }^{2}$ J. W. Gibbs, Elementary Principles in Statistical Mechanics, Collected Works (Yale University Press, New Haven, 1948); R. B. Lindsay and H. Margenau, Foundations of Physics (Dover, New York, 1957).

${ }^{3}$ C. Beck and E. G. D. Cohen, Physica A 322, 267 (2003).

${ }^{4}$ Nonextensive Entropy: Interdisciplinary Applications, edited by M. Gell-Mann and C. Tsallis (Oxford University Press, Oxford, 2004) and references therein; A. R. Plastino and A. Plastino, Phys. Lett. A 193, 140 (1994).

${ }^{5}$ G. Kaniadakis, M. Lissia, and A. Rapisarda (eds.), Physica A 305, Issues 1-2, pp. 1-350 (1 March 2002) and references therein.

${ }^{6}$ A. R. Plastino and A. Plastino, Phys. Lett. A 193, 251 (1994).

${ }^{7}$ M. Jauregui and C. Tsallis, "New representations of $\pi$ and Dirac delta using the nonextensive-statistical-mechanics $q$-exponential function," J. Math. Phys. 51, 063304 (2010).

${ }^{8}$ N. Goldenfeld, Lectures on Phase Transitions and the Renormalization Group (Addison-Wesley, New York, 1992).

${ }^{9}$ W. Rudin, Functional Analysis, 2nd ed. (McGraw-Hill, 1991).

${ }^{10}$ I. S. Gradshteyn and I. M. Ryzhik, Table of Integrals, Series, and Products, A. Jeffrey and D. Zwillinger (eds.), 7th ed. (Academic Press, Feb. 2007).

${ }^{11}$ We could extend the result to slowly increasing functions, but we do not need this refinement here. 